Open Access

\title{
Pancreatic Pseudocyst after Endoscopic Ultrasound-Guided Fine Needle Aspiration of Pancreatic Mass
}

\author{
Kwang Hyun Chung, Ji Kon Ryu, Hong Sang Oh, Ji Yeon Seo, \\ Eunhyo Jin, Dong Hyeon Lee, Yong-Tae Kim and Yong Bum Yoon \\ Department of Internal Medicine, Seoul National University College of Medicine, Seoul, Korea
}

\begin{abstract}
Endoscopic ultrasound-guided fine needle aspiration (EUS-FNA) is well known as a safe diagnostic procedure. We report the first case of pancreatic pseudocyst after EUS-FNA of the pancreatic body mass. A 60-year-old male underwent EUS-FNA for incidentally detected pancreatic solid mass which was suspected as neuroendocrine tumor. Two weeks later, the patient visited emergency room with acute abdominal pain and right upper quadrant tenderness; leukocytosis and elevated C-reactive protein, amylase, and lipase levels were noted. Computed tomography discovered newly developed $11.5 \times 9.5 \mathrm{~cm}$ sized cystic mass communicating with the main pancreatic duct. Cyst fluid analysis revealed amylase level of 3,423 U/L and fluid culture isolated Streptococcus parasanguinis. The cystic mass corresponds with pancreatic pseudocyst. FNA induced main pancreatic duct injury and fluid leakage may cause it. Endoscopists who perform EUS-FNA must remember that pancreatic main duct injury can occur as one of severe complications and that it could be treated successfully with endoscopic internal drainage.
\end{abstract}

Key Words: Pancreatic pseudocyst; Endosonography; Fine-needle biopsy; Complications

\section{INTRODUCTION}

Endoscopic ultrasound-guided fine needle aspiration (EUS-FNA) is well known as a useful diagnostic technique for pancreatic masses. Recent studies have reported high sensitivity, specificity and diagnostic accuracy of EUS-FNA. Meanwhile, the complications after EUS-FNA for pancreatic mass include abdominal pain, nausea and diarrhea, which are generally known as mild. ${ }^{1,2}$ Severe complications such as bacteremia, acute pancreatitis, hemorrhage, duodenal perforation, and aspiration pneumonia which require specific treatment are rarely reported. ${ }^{3-6}$ Although there is a report of pancreatic leakage with symptomatic ascites after EUS-FNA, there are few reports about pancreatic pseudocyst associated with EUS-FNA. ${ }^{7}$ In this report, we present the first case of Received: March 6, 2012 Revised: May 4, 2012 Accepted: July 4, 2012

Correspondence: Ji Kon Ryu

Division of Gastroenterology, Department of Internal Medicine, Seoul National University College of Medicine, 101 Daehak-ro, Jongno-gu, Seoul 110-744, Korea

Tel: +82-2-2072-1962, Fax: +82-2-762-9662, E-mail: jkryu@snu.ac.kr

(c) This is an Open Access article distributed under the terms of the Creative Commons Attribution Non-Commercial License (http://creativecommons.org/ licenses/by-nc/3.0) which permits unrestricted non-commercial use, distribution, and reproduction in any medium, provided the original work is properly cited. pancreatic pseudocyst after performing EUS-FNA at the pancreatic body solid mass.

\section{CASE REPORT}

A 60 -year-old male who had been followed for about 10 years for alcoholic fatty liver and hepatic hemangioma was referred for a newly detected pancreatic body mass on abdominal computed tomography (CT) scan. The mass was $2.4 \times 2.2$ $\mathrm{cm}$ and slightly enhanced. Main pancreatic duct was compressed by the mass and both proximal and distal side of the mass was dilated (Fig. 1). For the pathologic diagnosis, EUSFNA was planned. Initially, radial EUS (SSD-alpha 10 Ultrasound System; Aloka Co., Ltd., Tokyo, Japan) (GF-UE 260; Olympus Co., Tokyo, Japan) revealed a well demarcated dumbbell shape, $2.4 \mathrm{~cm}$-sized mass at the pancreatic body with peripheral hypoechogenecity and central hyperechogenecity. Under suspicion of neuroendocrine tumor, FNA was performed via transgastric approach with linear EUS (GFUCT240; Olympus Co.) and three passes were made with 22gauge needle (EchoTip Ultra, ECHO-22; Cook Endoscopy, Winston-Salem, NC, USA) (Fig. 2). No abnormal sign or symptom was noticed with the patient during and immediately af- 

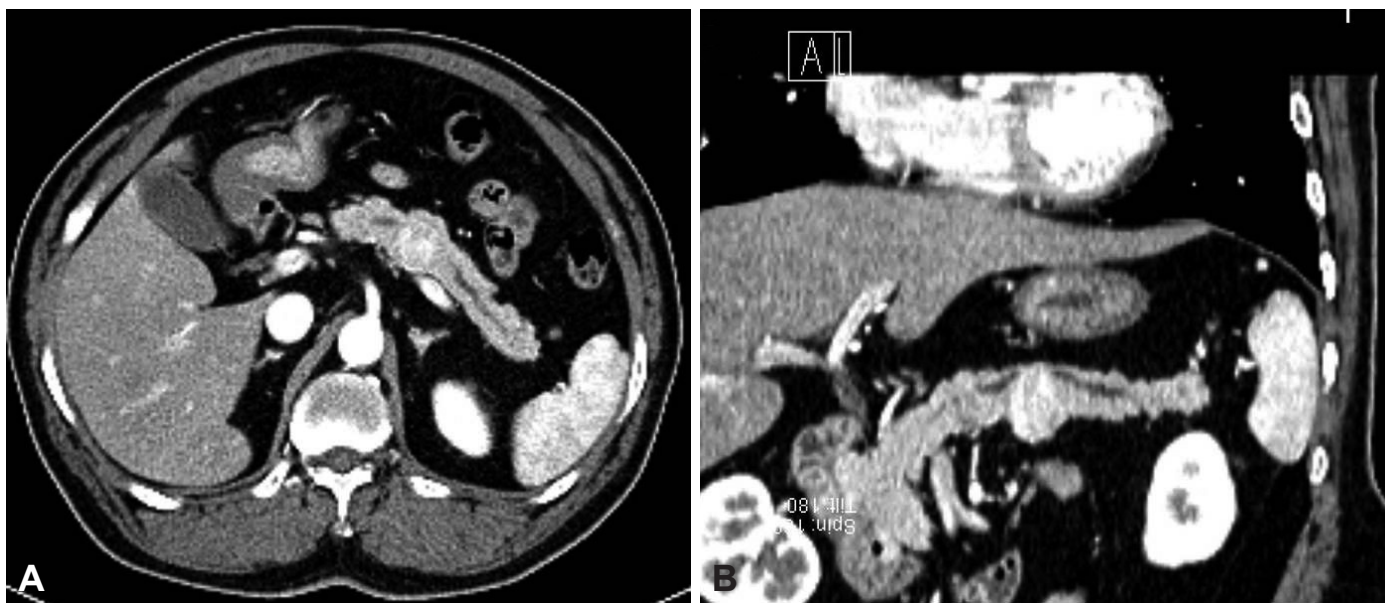

Fig. 1. Initial contrast enhanced abdominal computed tomography scan. (A) Transverse section. (B) Coronal reconstruction image. There are $2.4 \times 2.2 \mathrm{~cm}$ sized slightly enhancing mass in the pancreas body portion. Main pancreatic duct of both proximal and distal side of the mass was dilated.

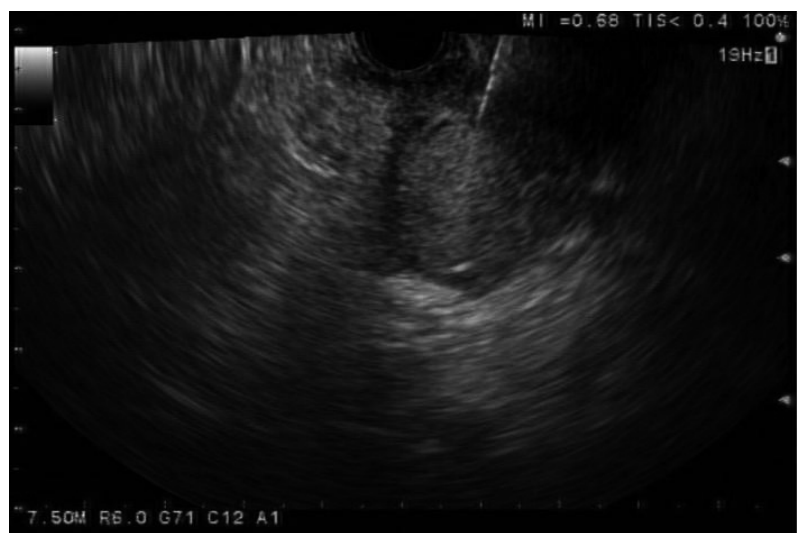

Fig. 2. Linear endoscopic ultrasound (GF-UCT240) which shows pancreatic body mass and passage of 22-gauge needle (EchoTip Ultra, ECHO-22). Fine needle aspiration was performed via transgastric approach and three passes were made.

ter the procedure.

The day after FNA, the patient complained about mild epigastric discomfort but he was discharged as scheduled. After discharge, abdominal discomfort exacerbated during the next 2 weeks and the patient eventually visited our emergency department. The physical examinations and routine blood tests including complete blood cell count, serum chemistry, and amylase/lipase were performed. The physical examinations revealed right upper quadrant tenderness and normoactive bowel sound. The leukocyte count was elevated to $14,700 /$ $\mathrm{mm}^{3}$ (range, 4,000 to 10,000) and C-reactive protein concentration was elevated to $18.19 \mathrm{mg} / \mathrm{dL}$ (range, 0 to 0.5 ). Amylase and lipase was also elevated to $668 \mathrm{U} / \mathrm{L}$ (range, 28 to 100) and $557 \mathrm{U} / \mathrm{L}$ (range, 22 to 51). Follow-up abdominal CT scan was performed and revealed a newly developed $11.5 \times 9.5 \mathrm{~cm}$ sized cystic mass. The mass was located between the posterior wall of gastric high body and pancreas, which has communication with the main pancreatic d uct (Fig. 3). Pancreatic body mass

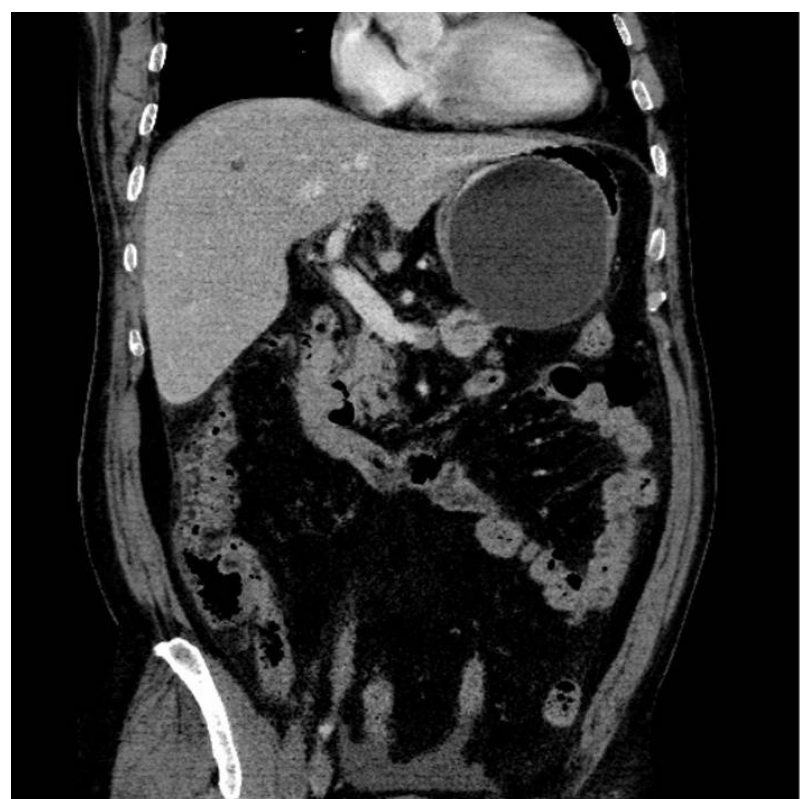

Fig. 3. Follow-up contrast enhanced abdominal computed tomography scan. A newly developed $11.5 \times 9.5 \mathrm{~cm}$ sized cystic mass is observed between the stomach and the pancreas. Communication is seen between the pseudocyst and the dilated pancreatic main duct.

was also seen with no interval change, and the pathologic report of aspiration cytology of the pancreatic body mass revealed many atypical cell clusters which was suspected as neuroendocrine tumor.

The cystic mass was assumed as pancreatic pseudocyst and internal drainage with endoscopic cystogastrostomy was planned. Radial EUS revealed bulging cystic mass just beside the gastric wall, and communication between the pancreas and the cyst was also presented. After confirming the absence of vascular structure between the puncture site and mass by EUS, cyst puncture was performed with needle knife (Hui- 
bregtse Single Lumen Needle Knife; Cook Endoscopy). Serosanguinous fluid was aspirated via puncture site and $10 \mathrm{Fr}, 5$ $\mathrm{cm}$ double pigtail plastic stent (Percuflex Amsterdam Singleuse Biliary Stents; Boston Scientific, Natick, MA, USA) was placed after dilation with hurricane balloon catheter. Cystic fluid analysis revealed the fluid amylase level of 3,423 U/L and carcinoembryonic antigen level of $4.7 \mathrm{mg} / \mathrm{mL}$, and Streptococcus parasanguinis was identified at the fluid culture. Endoscopic retrograde cholangiopancreatography was also performed for internal drainage of the pseudocyst. Pancreatogram showed the leakage of dye from the pancreatic duct of the body portion, and transpapillary pancreatic duct stenting using 7 Fr, $15 \mathrm{~cm}$ straight plastic stent (PercuflexTM Amsterdam Single-use Biliary stents; Boston Scientific) was performed across the disrupted pancreatic duct to promote pancreatic duct healing (Fig. 4). Soon after, the fever and abdominal pain were gradually subsided and the condition of the patient was stabilized.

On the fifth day of the drainage procedure, distal pancreatectomy was performed for resection of the neuroendocrine tumor. Simultaneously, cholecystectomy, and surgical drainage of the pseudocyst were performed without acute operation related complication. Pathologic report confirmed the mass as a neuroendocrine tumor of grade 2 and the resected cyst as a pancreatic pseudocyst with adjacent fat necrosis (Fig. 5).

\section{DISCUSSION}

EUS-FNA is generally considered as a safe procedure with extremely low frequency of major complications. Acute pancreatitis could occur after performing EUS-FNA; however, most cases are self-limiting mild cases and no EUS-FNA related pseudocyst development has been reported. ${ }^{6,8}$ To our
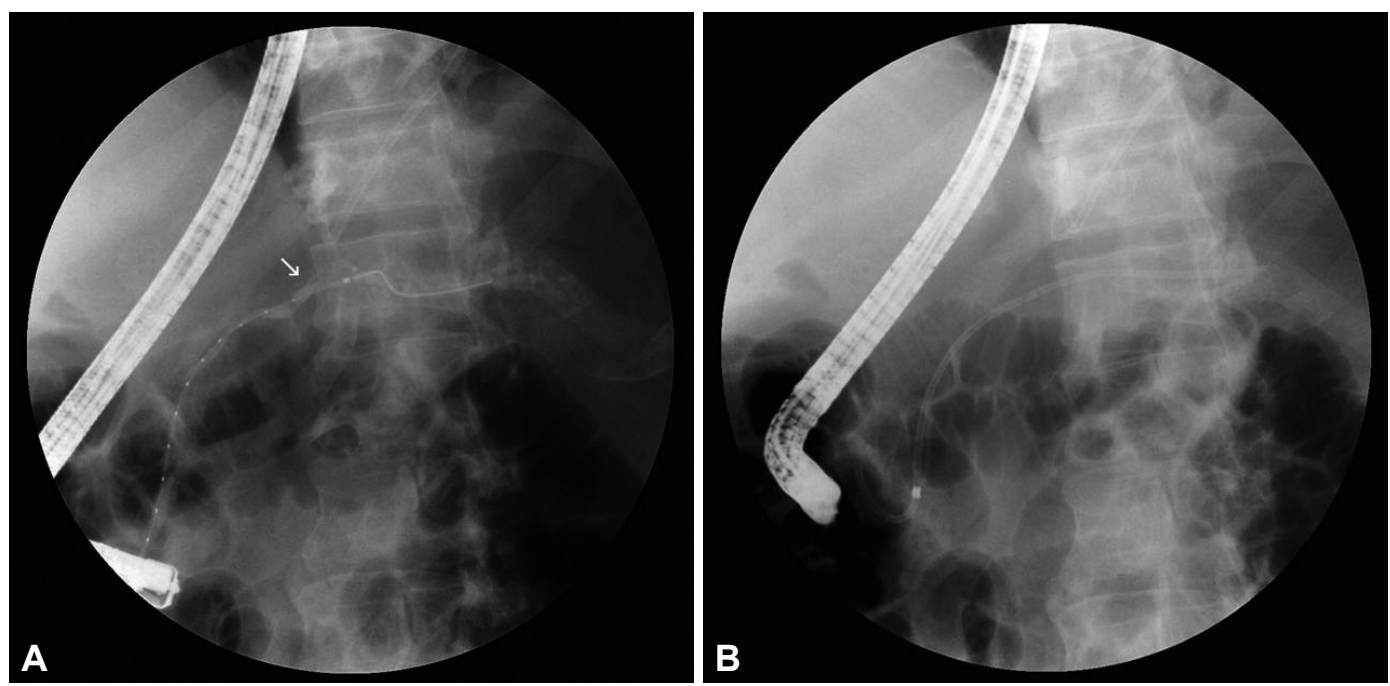

Fig. 4. Endoscopic retrograde pancreatogram. (A) Pancreatogram showed the leakage of dye from the pancreatic duct of the body portion (white arrow), and (B) transpapillary internal drainage using $7 \mathrm{Fr}, 15 \mathrm{~cm}$ straight plastic stent (Percuflex Amsterdam Single-use Biliary Stents) was performed.
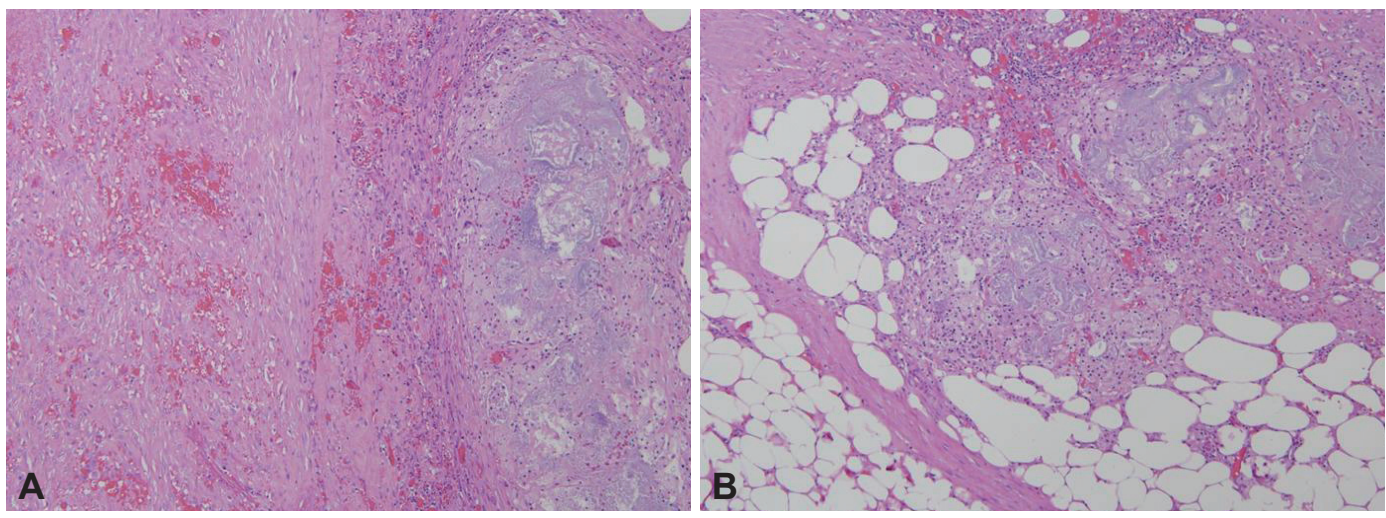

Fig. 5. (A) Histological image of the resected pancreatic mass showing pseudocyst and organizing hematoma in the cyst cavity (H\&E stain, $\times 400)$. (B) Adjacent fat necrosis was also seen (H\&E stain, $\times 400)$. 
knowledge this is the first reported case of a pancreatic pseudocyst as a complication of EUS-FNA.

In this case, cystic mass was developed within 2 weeks after the EUS-FNA and analysis of the aspirated cyst fluid showed elevated amylase level over 5 times of the serum amylase level. There were no any other causative factors that could induce acute pancreatitis and pseudocyst within 2 weeks. This makes EUS-FNA as the most likely etiology of the pseudocyst. Moreover, communication between the cystic mass and the pancreas which was located at the site of previous needle passage strengthen the suspicion. Presumed mechanism of pseudocyst development is FNA induced pancreatic duct injury and leakage of the pancreatic fluid into the peritoneal cavity. At the initial CT, main pancreatic duct was slightly compressed by the pancreatic mass and distal duct dilatation was also showed. Main pancreatic duct stenosis and increased distal pressure might have instigated the pancreatic fluid leakage. Interestingly, the main pancreatic duct on the proximal side of the pancreatic mass also showed a similar degree of dilatation, as shown in Fig. 1. The cause of proximal side dilatation was not clear; however, considering that the patient was a chronic alcoholic, we speculated that the patient had chronic pancreatitis previously and pancreatic duct dilatation as a result.

Although there was no report about EUS-FNA associated pseudocyst, there was a report of pancreatic leakage after EUSFNA. In that case, the patient was managed by transpapillary pancreatic duct stenting. ${ }^{7}$ Likewise, our patient was also managed successfully with endoscopic drainage. We chose endoscopic cystogastrostomy as initial therapy followed by transpapillary stenting, because the size of pseudocyst exceeded 6 $\mathrm{cm}$ and the clinical symptoms of the patient were severe. ${ }^{9}$

In conclusion, Endosonographists who perform EUS-FNA must keep in mind that pancreatic main duct injury can occur as one of severe complications-especially in a case showing proximal stenosis and distal duct as well as main pancreatic duct dilatations. It can be managed by endotherapy such as transpapillary or transmural stenting.

\section{Conflicts of Interest}

The authors have no financial conflicts of interest.

\section{REFERENCES}

1. Itoi T, Sofuni A, Itokawa F, Irisawa A, Khor CJ, Rerknimitr R. Current status of diagnostic endoscopic ultrasonography in the evaluation of pancreatic mass lesions. Dig Endosc 2011;23 Suppl 1:17-21.

2. Yoshinaga S, Suzuki H, Oda I, Saito Y. Role of endoscopic ultrasoundguided fine needle aspiration (EUS-FNA) for diagnosis of solid pancreatic masses. Dig Endosc 2011;23 Suppl 1:29-33.

3. O’Toole D, Palazzo L, Arotçarena R, et al. Assessment of complications of EUS-guided fine-needle aspiration. Gastrointest Endosc 2001;53: 470-474.

4. Carrara S, Arcidiacono PG, Mezzi G, Petrone MC, Boemo C, Testoni PA. Pancreatic endoscopic ultrasound-guided fine needle aspiration: complication rate and clinical course in a single centre. Dig Liver Dis 2010;42:520-523.

5. Williams DB, Sahai AV, Aabakken L, et al. Endoscopic ultrasound guided fine needle aspiration biopsy: a large single centre experience. Gut 1999;44:720-726.

6. Eloubeidi MA, Tamhane A, Varadarajulu S, Wilcox CM. Frequency of major complications after EUS-guided FNA of solid pancreatic masses: a prospective evaluation. Gastrointest Endosc 2006;63:622-629.

7. Reddymasu S, Oropeza-Vail MM, Williamson S, Jafri F, Olyaee M. Pancreatic leak after endoscopic ultrasound guided fine needle aspiration managed by transpapillary pancreatic duct stenting. JOP 2011; 12:489-490.

8. Wang KX, Ben QW, Jin ZD, et al. Assessment of morbidity and mortality associated with EUS-guided FNA: a systematic review. Gastrointest Endosc 2011;73:283-290.

9. Samuelson AL, Shah RJ. Endoscopic management of pancreatic pseudocysts. Gastroenterol Clin North Am 2012;41:47-62. 